\title{
Una propuesta didáctica mediante el Robot Nao para la enseñanza del idioma inglés como una herramienta de apoyo para la educación 4.0
}

\section{A didactic proposal through the Nao robot for teaching the English language as support tool for education 4.0}

\author{
MANCILLA, Víctor†, HUERTA, Patricia, TREJO, Alejandra y CARRANZA, Cielo \\ Universidad Politécnica de Juventino Rosas, Departamento de Ingeniería en Redes y Telecomunicaciones.
}

ID $1^{\mathrm{er}}$ Autor: Víctor, Mancilla / ORC ID: 0000-0003-0020-2174

ID $1^{\text {er }}$ Coautor: Patricia, Huerta / ORC ID: 0000-0002-2708-5046

ID 2do Coautor: Alejandra, Trejo / ORC ID: 0000-0003-1582-1971

ID $3^{\text {er }}$ Coautor: Cielo, Carranza / ORC ID: 0000-0001-7896-5054

DOI: $10.35429 / J O T E .2020 .11 .4 .12 .16$

Recibido Enero 15, 2020; Aceptado Marzo 31, 2020

\begin{abstract}
Resumen
NAO es un robot humanoide desarrollado por la empresa SoftBank Robotics (antes llamada Aldebaran Robotics). NAO permite la interactividad con sus componentes, convirtiéndolo en una plataforma versátil para el desarrollo de aplicaciones en distintos sectores, como salud, educación e investigación. El presente trabajo titulado "Una propuesta didáctica mediante el Robot Nao para la enseñanza del idioma inglés como una herramienta de apoyo para la educación 4.0." tiene como objetivo principal diseñar, desarrollar e implementar situaciones de aprendizaje que permitan incrementar el nivel de expresión y comprensión del idioma inglés, mediante el robot humanoide Nao, quien ayudara a realizar actividades que les permitan a las personas interactuar con el robot esto primeramente para que la personas no se cohíban al hablar este idioma. El proyecto está elaborado para enseñar inglés a niños y jóvenes en edad escolar pertenecientes a una institución educativa, con ayuda del Robot Humanoide Nao perteneciente al área de robótica educativa de la Universidad Politécnica de Juventino Rosas. Para el diseño de las actividades a realizar, se investigó los temas de gramática que se imparten en los niveles de escolaridad desde primaria hasta universidad para poder lograr el diseño que se necesita en cada nivel educativo y así el Robot Humanoide Nao pueda realizar su tarea de manera correcta dichos temas pertenecen al plan de estudios diseñados por la SEP (secretaria de Eduacación Pública).
\end{abstract}

\begin{abstract}
NAO is a humanoid robot developed by SoftBank Robotics (formerly Aldebaran Robotics). NAO allows interactivity with its components, making it a versatile platform for the development of applications in different sectors, such as health, education and research. The present work entitled "A didactic proposal through the Nao Robot for teaching English as a support tool for education 4.0." Its main objective is to design, develop and implement learning situations that increase the level of expression and understanding of the English language, through the humanoid robot Nao, who will help to carry out activities that allow people to interact with the robot first so that people do not shy away from speaking this language. The project is designed to teach English to children and young people of school age belonging to an educational institution, with the help of the Nao Humanoid Robot belonging to the area of educational robotics of the Polytechnic University of Juventino Rosas. For the design of the activities to be carried out, the grammar topics that are taught in the levels of schooling from primary to university were investigated to be able to achieve the design that is needed at each educational level and so the Nao Humanoid Robot can perform its task of Correctly, these topics belong to the curriculum designed by the SEP (Secretary of Public Education).
\end{abstract}

Robot nao, Interactuar, Enseñanza del inglés, Educación 4.0

Nao robot, interact, teaching English Education 4.0

Citación: MANCILLA, Víctor, HUERTA, Patricia, TREJO, Alejandra y CARRANZA, Cielo. Una propuesta didáctica mediante el Robot Nao para la enseñanza del idioma inglés como una herramienta de apoyo para la educación 4.0. Revista de Educación Técnica. 2020. 4-11:12-16.

$\dagger$ Investigador contribuyendo como primer autor. 


\section{Introducción}

La robótica educativa para la enseñanza del idioma inglés como una herramienta de apoyo para la educación 4.0, mediante situaciones de aprendizaje ayudará a niños y jóvenes en edad escolar pertenecientes a una institución educativa a incrementar su speaking y listening el cual será medido a través del diseño experimental en el que se mostrarán los resultados finales que permitirán confirmar la hipótesis del Proyecto.

Este proyecto tiene como objetivo diseñar, desarrollar e implementar situaciones de aprendizaje que permitan mediante la robótica educativa utilizando la plataforma robot $\mathrm{Nao}$ el incremento del nivel de expresión y compresión del idioma inglés. Haciendo uso de las tecnologías, se pretende incrementar los índices de aprendizaje en este idioma, con la robótica educativa sirviendo como una herramienta pedagógica para dar un inicio a la educación 4.0 dentro de las instituciones en el municipio de Santa Cruz de Juventino Rosas.

Lo que buscamos con este Proyecto es que México incremente sus habilidades en el idioma inglés ayudando a mejorar su posición en el ranking en EF English Proficiency Index. En el cual "México se encuentra en el lugar \#67 con un nivel bajo" (EF English Proficiency Index (EPI), 2019).

La opción de enseñar el idioma inglés a través de la robótica educativa es debido a que los robots "crean fácilmente un vínculo de empatía con estudiantes, profesores e investigadores por sus llamativas apariencias, tamaños moderados y comportamientos humanoides" (Aldebaran Softbank, 2020).

El robot a utilizar es el robot nao quien "es un robot versátil y de alto rendimiento para satisfacer las expectativas de profesores e investigadores. Es una plataforma avanzada para el estudio en profundidad, como la interacción hombre-máquina, la computación cognitiva y la navegación autónoma, etc." (Aldebaran Softbank, 2020).

\section{Secuencia de aprendizaje}

Una situación de aprendizaje implica la realización de un conjunto de actividades articuladas que los estudiantes llevarán a cabo para lograr ciertos fines o propósitos educativos en un lapso y en un contexto específicos, lo que supone distintos tipos de interacciones:

- Con los integrantes del grupo y personas externas.

- Con información obtenida de diversas fuentes: bibliografía, entrevistas, observaciones, videos, etc.

- En diversos tipos de espacios o escenarios: aula, laboratorio, taller, empresas, instituciones, organismos, obras de construcción, etc.

Es deseable que estas situaciones de aprendizaje se vinculen a situaciones reales del ámbito social o profesional en las que tienen lugar acontecimientos, hechos, procesos, interacciones, fenómenos... cuya observación y análisis resultan relevantes para adquirir aprendizajes o en las que se pueden aplicar los aprendizajes que van siendo adquiridos a lo largo del curso (Instituto Tecnológico y de Estudios SuperioreS, 2019).

En este Proyecto se usaron las situaciones de aprendizajes mediante la robótica educativa para generar el interés de la persona , a través de las situaciones de aprendizaje que ayudaran a las personas a adquirir habilidades que beneficien su pronunciación, así como la habilidad auditiva en el idioma inglés, esto debido a que el robot se dirige a las personas en este idioma por consecuencia el robot ayudara a la persona a incrementar esta habilidad para poder interactuar con el robot, así mismo la pronunciación es la habilidad del habla que este robot ayudara a mejorar en las personas generando confianza al expresarse con el robot y poder adquirir una mayor fluidez en el momento de hablar el idioma inglés.

Otra de las características de este robot o plataforma es la de poder integrar la inteligencia artificial (IA). Para generar planes de aprendizaje personalizados, el costo de esta plataforma puede oscilar depende del proveedor, el hardware y software requerido. 
Un ejemplo de esto puede ser que se compre solo la cabeza del robot y con este hardware se puede empezar a trabajar y el costo se reduce en gran medida, otra propuesta es trabajar de manera virtual mediante software como Choregraphe o Webots los cuales tienen una versión gratuita.

\section{Diseños experimentales}

En la investigación de enfoque experimental el investigador manipula una o más variables de estudio, para controlar el aumento o disminución de esas variables y su efecto en las conductas observadas. Dicho de otra forma, un experimento consiste en hacer un cambio en el valor de una variable (variable independiente) y observar su efecto en otra variable (variable dependiente). Esto se lleva a cabo en condiciones rigurosamente controladas, con el fin de describir de qué modo o por qué causa se produce una situación o acontecimiento particular. Los métodos experimentales son los adecuados para poner aprueba hipótesis de relaciones causales (Leon Rodrigo, y otros, 2015. consultado en febrero 2020.).

\begin{tabular}{|l|l|l|}
\hline \multicolumn{2}{|c|}{ Fase } & \multicolumn{1}{|c|}{$\begin{array}{c}\text { Grupo experimental } \\
\text { control }\end{array}$} \\
\hline Experimental & $\begin{array}{l}\text { Exposición al factor } \\
\text { experimental }\end{array}$ & $\begin{array}{l}\text { No } \\
\text { exposición } \\
\text { al factor }\end{array}$ \\
\hline De medición & Medida 1 & Medida 2 \\
\hline De análisis & Comparación de resultados \\
\hline
\end{tabular}

Tabla 1 Proceso para el análisis de diseño experimental Fuente: elaboración propia [Word]

Para poder entender mejor este tipo de diseño, vamos a enfocarlo al objetivo de este proyecto que es, mejorar el rendimiento académico de un grupo de alumnos en la materia de inglés, de manera que el profesor utilizará la robótica como una herramienta de apoyo para la enseñanza del idioma principalmente para mejor la expresión de sus alumnos (Speaking). Para realizar este tipo de diseño experimental, tomaremos dos grupos de alumnos homogéneos y, uno de ellos, que será el grupo experimental, aprenderá la materia por medio de la robótica, mientras que el grupo control seguirá con la metodología de siempre, es decir, la explicación del profesor. En este tipo de diseño, se comenzará aplicando una prueba previa de rendimiento, es decir, un pretest, a ambos grupos, usando ambos la explicación del profesor como medio de aprendizaje.
Tras hacer esto, al finalizar la unidad didáctica se les aplicará a ambos grupos otra evaluación que es un postest para comprobar las diferencias habidas en el rendimiento académico.

\begin{tabular}{|l|l|l|l|}
\hline Grupo & Pretest & Tratamiento & Postest \\
\hline A & SI & SI & SI \\
\hline B & SI & NO & SI \\
\hline
\end{tabular}

Tabla 2 Diseño experimental del proyecto Fuente: elaboración propia [word]

Como Podemos observar en la tabla 1, estos diseños se constituyen por dos grupos. Por una parte, tenemos el grupo experimental, que es el que recibirá el estímulo y, por otro lado, tendremos un grupo control, que nos servirá como punto de referencia para apreciar las variaciones que se produzcan en el grupo control, por ello es importante que ambos grupos sean lo más parecidos posibles. Al finalizar la unidad, se le hará una prueba de evaluación de rendimiento (postest) a ambos grupos a través del cual se podrán ver las diferencias habidas tras el experimento. Este diseño es de los más completos que se pueden usar en investigación experimental, pues al incorporar un grupo de control que tiene las mismas experiencias que el grupo experimental, excepto el tratamiento, la validez interna queda asegurada.

\section{Temas que se abordan en las secuencias de aprendizaje}

Mediante la plataforma Robot Nao, se estructurarán secuencias de aprendizaje donde se aborden, los siguientes temas como son; Countries and nationalities, word stress, possessive's, simple present, questins and short answers, daily activities, present continuous. Dónde estás secuencias de aprendizajes ayuden a generar las siguientes habilidades de lenguaje como: pronunciación (pronunciation), gramática (grammar), auditiva (listening).

\section{Diseño del pretest}

El pretest fue desarrollado para medir el conocimiento que tienen hasta el momento las personas (grupo A y grupo B), dichos resultados de esta prueba ayudaran a medir el avance de mejora que obtuvieron las personas de ambos grupos después del tratamiento o proceso de pruebas que se medirá a través de una segunda evaluación (protest).

MANCILLA, Víctor, HUERTA, Patricia, TREJO, Alejandra y CARRANZA, Cielo. Una propuesta didáctica mediante el Robot Nao para la enseñanza del idioma inglés como una herramienta de apoyo para la educación 4.0. Revista de Educación Técnica. 2020 


\section{Secuencias de aprendizaje para nivel básico}

En esta secuencia de aprendizaje que se desarrolló en la primera etapa del proyecto se abordaron los temas básicos para comprender y mejorar las habilidades de las personas con esta primera secuencia de aprendizaje donde lo primero que se explica es el verbo To $\mathrm{Be}$, nacionalidades, números, profesiones, saludos y despedidas lo que les permite a las personas poder realizar una presentación acerca de si misma.

La muestra que se tiene proyectada es la del $50 \%$ de la matrícula de inglés en la carrera de ingeniería en redes y telecomunicaciones que son alrededor de 100 alumnos.

\section{Pruebas}

\section{Diseño del protest}

El protest es la evaluación con la cual se medirá el avance de las personas después de estar en el proceso de pruebas o tratamiento así mismo se aplicará el protest al grupo que seguirá trabajando con la metodología del docente esto para realizar la comparación y poder comparar el progreso que obtuvieron ambos grupos y con ello poder comprobar la Hipótesis de este proyecto.

\section{Resultados}

Al aplicarse las secuencias y los instrumentos de medición como lo son el pretest y protest observamos los siguientes resultados.

Como se puede observar en la figura 1. Los alumnos están motivados y sorprendidos utilizando nuestro instrumento pedagógico, que en este caso es la robótica educativa mediante la plataforma robot NAO, se observa la interacción hombre maquina y el uso de las tecnologías aplicadas a la educación 4.0.

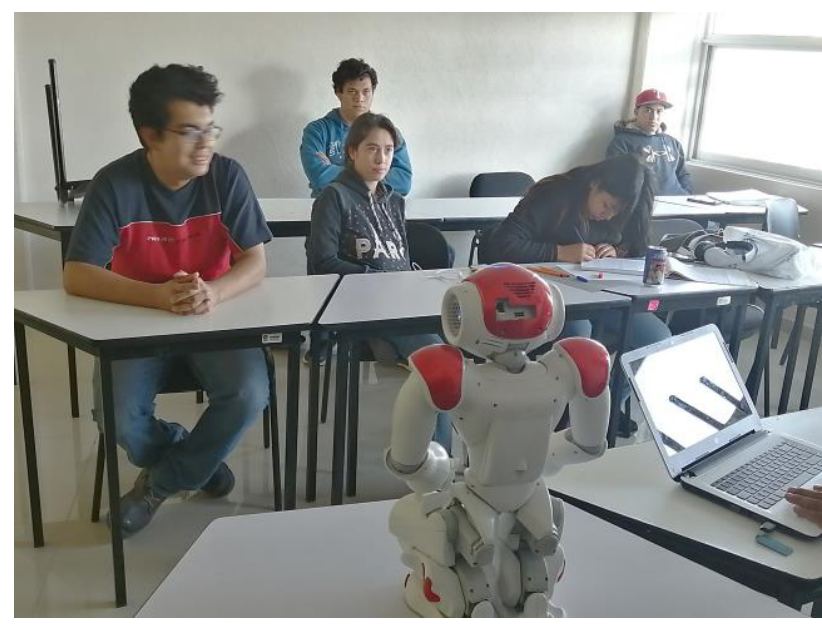

Figura 1 Puesta en marcha pretest

Fuente: elaboración propia [cam]

En otro de los resultados obtenidos mediante nuestra metodología aplicando el protest observamos como los alumnos muestran un avance en la pronunciación del idioma inglés, porque el robot NAO solo reconoce las palabras pronunciadas mínimo con un $50 \%$. por lo tanto, si no llegan a este porcentaje el robot no interactuara con el alumno. Como se muestra en la figura 2 .

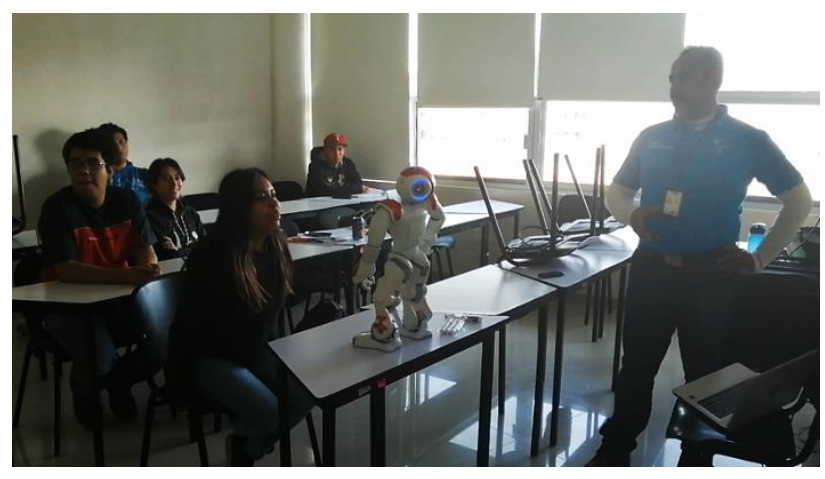

Figura 2 Puesta en marcha protest

Fuente: elaboración propia [cam]

\section{Conclusiones}

Durante el desarrollo de la propuesta se observó que el idioma ingles es una de las materias más importantes en cualquier institución educativa, pero también es una de las materias con mayor índice de reprobación, es por eso que a través de la robótica educativa pretendemos incrementar el índice de comprensión y expresión de idioma, esto lo hacemos con el fin de que en un futuro los niños y los jóvenes que se encuentran en su formación académica puedan abrirse más puertas en su ámbito laboral, ya que este idioma es uno de los pilares más importantes para poder obtener un buen trabajo. 
Esta propuesta nos permitirá lograr en los alumnos incrementar y adquirir competencias educativas y laborales que se necesitan en la educación 4.0 y en la industria 4.0

\section{Referencias}

Aldebaran Softbank, R. (12 de ENERO de 2020). Pepper y NAO, robots para la educación. Obtenido de Robotics, SoftBank: https://www.softbankrobotics.com/emea/en/pep per-and-nao-robots-education

EF English Proficiency Index (EPI), E. (30/noviembre/2019 de AGOSTO de 2019). Índice del EF English Proficiency. Obtenido de Índice del EF English Proficiency: https://www.ef.com.mx/epi/

Instituto Tecnológico y de Estudios SuperioreS, I. (enero de 2019). Universidad Jesuita de Guadalajara. Obtenido de Occidente, Instituto Tecnológico y de Estudios Superiores de

Leon Rodrigo, I., García Gordo, E., Gil Álvaro , B., Ríos Brea , L., Serrano, , A., \& García Sanz, L. (2015. consultado en febrero 2020.). Métodos de investigacion de enfoque experimental. redalyc, 1(1):20-27. 